\title{
REGULARITY OF THE ALBANESE MAP FOR NONORIENTABLE SURFACES
}

\author{
WILLIAM H. MEEKS, III
}

The function theory of a closed Riemann surface is fundamentally related to the Jacobi or Albanese map of the Riemann surface into a complex torus called the Jacobi variety of the surface. The Jacobi embedding theorem [2] states that this natural map is a smooth embedding and Abel's theorem [1] gives necessary and sufficient conditions, in terms of this mapping, for a divisor of the Riemann surface to be the divisor of a meromorphic function. Other important conformal information about the Riemann surface can be obtained from the embedding into its Jacobi variety. For example, Torelli's theorem [3] which describes the moduli space of Riemann surfaces in terms of the Jacobi map displays the deep interplay between the conformal structure of the Riemann surface and its Jacobi mapping.

The Albanese map, which in the case of Riemann surfaces is usually called the Jacobi map, is a holomorphic map of the Riemann surface into a flat complex torus, and this map induces an isomorphism between the first homology groups of the surface and the complex torus. In particular, the Albanese map is a harmonic map between the Riemann surface and a flat torus. For any closed Riemannian manifold $M$ one can define a natural harmonic map $f: M \rightarrow A(M)$ where $A(M)$ is a flat torus $\mathrm{R}^{n} / \Lambda$ for some lattice $\Lambda$. The map $f$ also has the property that it induces an isomorphism between first integral homology of $M$ (modulo torsion) and the first integral homology group of $A(M)$. The map $f$ is called the Albanese map of $M$ and $A(M)$ is called the Albanese variety of $M$. A rigorous definition of $f: M \rightarrow A(M)$ is given at the beginning of the main body of the paper. For the moment it suffices to note that the Albanese map satisfies the following universal property: If $g: M \rightarrow T=\mathbf{R}^{n} / L$ is a harmonic map with $g\left(p_{0}\right)=0$, and $f\left(p_{0}\right)=0$, then $g=\pi \circ f$ for some "linear" homomorphism $\pi: A(M) \rightarrow T$.

Received October 8, 1987. This research was partially supported by the National Science Foundation and Department of Energy Grants NSF-DMS-8611574 and DE-FG02-86ER 25015. 
In this paper we prove a theorem on the regularity of the Albanese map of a nonorientable Riemannian surface. This theorem, Theorem 1.4, gives necessary and sufficient conformal conditions for the Albanese map of a nonorientable Riemannian surface to be a smooth embedding. The proof of this theorem is based on classical results in Riemann surface theory. These results include Abel's theorem and the embedding of the canonical curve of a nonhyperelliptic Riemannian surface.

A surface $M$ in $\mathbf{R}^{n}$ is called $n$-periodic if there exists a lattice $L_{M}$ of $\mathbf{R}^{n}$ such that $M$ is invariant under translation by elements of $L_{M}$. In classical differential geometry the collection of $n$-periodic minimal surfaces $\mathbf{R}^{n}$ forms a rich and important collection of minimal surfaces. For example, the lifts of Riemann surfaces in their Jacobi varieties to $\mathbf{C}^{n}$ are important examples of $2 n$-periodic least-area minimal surfaces in $\mathbf{R}^{2 n}$. Of special geometric interest is the case of 3-periodic minimal surfaces in $\mathbf{R}^{3}$.

In his thesis, Meeks [4] described a six-dimensional family of noncongruent embedded minimal surfaces of genus 3 in flat 3-tori. Recently [6] he has shown that ever flat 3-torus contains an infinite number of the surfaces in this family via an independent minimax argument. What Meeks actually proves in [6] is a lemma which shows that every flat 3-torus $T$ contains an infinite number of branched nonorientable minimal surfaces $f_{i}: M_{i} \rightarrow T$ such that $f_{i}$ is the Albanese map of a nonorientable "Riemann surface" $M_{i}$ with Euler characteristic -2 . These branched minimal surfaces arise from a variational problem which yields solutions which are branched "conformal" harmonic maps rather than smooth embeddings. However, it follows from the regularity theorem (Theorem 1.4) in the present paper that the branched immersions $f_{i}: M \rightarrow T$ are smooth embeddings.

Applying the techniques in [7] one can prove that every flat 4-torus contains an infinite number of branched minimal immersions of nonorientable surfaces of Euler characteristic -3 . Our regularity theorem and Corollary 2 imply that these surfaces are smooth embeddings and that they lift to properly embedded 4-periodic nonorientable minimal surfaces in $\mathbf{R}^{4}$.

In a natural sense the regularity theorem for the Albanese map of a nonorientable surface (Theorem 1.4) gives a complete generalization of the Jacobi embedding theorem for Riemann surfaces to the case of nonorientable Riemannian surfaces. So far the only application of this regularity theorem has been to prove the existence of many $n$-periodic smoothly embedded minimal surfaces in $\mathbf{R}^{n}$. However it seems likely that this regularity theorem and similar theorems might shed some information on the geometry of the classical case of a Riemann surface contained in its Jacobi variety. For example, 
as shown in [7], a nonhyperelliptic Riemann surface contains, up to translation, two different embeddings in its Jacobi variety where each embedding represents the limit of a continuous one-parameter family of nonorientable harmonic surfaces.

The author would like to thank Professor Gaber at Institut des Hautes Études Scientifiques for some informative discussions of the material contained in this paper.

\section{The regularity theorem}

We first give a rigorous definition of the Albanese map for a closed Riemannian manifold.

Definition 1.1. Let $M$ be a closed Riemannian manifold and let $h_{1}, \cdots, h_{k}$ be a basis for the vector space of harmonic 1 -forms on $M$. Let $p_{0}$ be a basepoint for $M$ and let

$$
\Lambda=\left\{\int_{\gamma}\left(h_{1}, \cdots, h_{k}\right)^{t} \mid \gamma \in H_{1}(M, Z)\right\} .
$$

Let $f: M \rightarrow A(M)=\mathbf{R}^{k} / \Lambda$ defined by the integral $f(p)=\left[\int_{p_{0}}^{p}\left(h_{1}, \cdots, h_{k}\right)^{t}\right]$. The flat torus $A(M)$ which depends on the choice of basis of the harmonic 1-forms is called the Albanese torus for $M$ and $f: M \rightarrow A(M)$ is called the Albanese map. The map $f$ is the unique harmonic map of $M$ into $A(M)$ in the homotopy class of $f$ up to translation in $A(M)$.

In classical Riemann surface theory a basis of harmonic forms can be chosen so that $h_{2 i}={ }^{*} h_{2 i-1}$ where ${ }^{*}$ is the Hodge star operator. In this case $\omega_{i}=h_{2 i-1}+\sqrt{-1}^{*} h_{2 i}$ is a holomorphic 1-form, $A(M)$ has a natural orthogonal almost complex "linear" structure so that the map $f: M \rightarrow A(M)$ is holomorphic, and much of the important conformal information of $M$ can be uncovered from the placement of $f(M)$ in $A(M)$. A key result in this direction is Abel's theorem which states that a necessary and sufficient condition for $\sum_{i=1}^{n}\left(p_{i}-q_{i}\right)$ to be a divisor of a meromorphic function on $M$ is

$$
\sum f\left(p_{i}\right)=\sum f\left(q_{i}\right) \text { in } A(M) .
$$

Furthermore it follows from Abel's theorem that $f$ is always injective. We now analyze the Albanese map for a nonorientable surface. The Albanese map for a nonorientable surface which is diffeomorphic to the projective plane is the constant map. Since harmonic 1-forms on a flat manifold are parallel, we note that the Albanese map of a Klein bottle is a submersion onto a circle.

Suppose that $M$ is a nonorientable closed Riemannian surface, $p_{0}$ is a base point for $M$ and $\left\{h_{1}, \cdots, h_{k}\right\}$ is a fixed basis for the harmonic 1-forms of $M$. 
Let $\pi: \tilde{M} \rightarrow M$ denote the oriented 2-sheeted cover of $M$, let $\sigma$ denote the order-two covering transformation and let $\tilde{h}_{i}=\pi^{*}\left(h_{i}\right)$ and $\omega_{i}=\tilde{h}_{i}+\sqrt{-1} * \tilde{h}_{i}$. Choose $\tilde{p}_{0}$ to be one of the points in $\pi^{-1}\left(p_{0}\right)$. Then $\left\{\omega_{1}, \cdots, \omega_{k}\right\}$ forms a basis for the holomorphic 1 -forms on $\tilde{M}$. Let $\tilde{f}: \tilde{M} \rightarrow A(\tilde{M})=\mathrm{C}^{k} / \tilde{\Lambda}$ and $f: M \rightarrow A(M)$ be the corresponding Albanese maps and let $\bar{\pi}: A(\tilde{M}) \rightarrow$ $A(M)$ be the linear homomorphism which is induced by the harmonic maps $f \circ \pi: \tilde{M} \rightarrow A(M)$. Let $T(\sqrt{-1})$ denote the subtorus of $A(\tilde{M})$ which is the image torus of the purely imaginary vectors in $\mathbf{C}^{k}$. Let $\mathbf{R e}: A(\tilde{M}) \rightarrow$ $A(\tilde{M}) / T(\sqrt{-1})=\hat{A}(\tilde{M})$ be the linear homomorphism to the torus $\hat{A}(\tilde{M})$ induced by the real projection $\mathbf{R e}: \mathbf{C}^{k} \rightarrow \mathbf{R}^{k}$ and let $H: \hat{A}(\tilde{M}) \rightarrow A(M)$ be the induced projection.

Lemma 1.2. Let $M$ be a closed nonorientable Riemannian surface. Then the following properties hold:

(1) $\sigma$ extends to a map $\bar{\sigma}=\varsigma v$ o $c$ of $A(\tilde{M})$, where $c$ is the map on $A(\tilde{M})$ induced by complex conjugation on $\mathrm{C}^{k}$ and $\varsigma v_{0}$ is translation by the point $v_{0}=\tilde{f}\left(\sigma\left(p_{0}\right)\right)=-c\left(v_{0}\right)$.

(2) $\sigma$ extends to a map $\tilde{\sigma}$ on $\hat{A}(\tilde{M})$ which is a pure translation by the point $\boldsymbol{\operatorname { R e }}\left(v_{0}\right)$.

(3) The map c: $A(\tilde{M}) \rightarrow A(\tilde{M})$ satisfies $c(x)=-x$ for all $x$ in the kernel of $\bar{\pi}$.

Proof. Let $h_{1}, h_{2}, \cdots, h_{k}$ be a basis for the vector space of harmonic 1forms of $M$. Since $\sigma$ is an orientation reversing isometry of the orientable surface $\tilde{M}$ and $\sigma$ leaves invariant the harmonic 1-forms $\tilde{h}_{i}=\pi^{*}\left(h_{i}\right)$ and $\sigma^{*}\left({ }^{*} \tilde{h}_{i}\right)=-{ }^{*} \tilde{h}_{i}$. Hence $\sigma^{*}\left(\omega_{i}\right)=\bar{\omega}_{i}$, where $\bar{\omega}_{i}$ denotes the complex conjugate to the form $\omega_{i}=\tilde{h}_{i}+\sqrt{-1}^{*} \tilde{h}_{i}$. A simple Euler characteristic calculation shows $\omega_{1}, \cdots, \omega_{k}$ is a basis for the holomorphic 1-forms on $\tilde{M}$. Now let $W=\left(\omega_{1}, \cdots, \omega_{k}\right)^{t}$. Then

$$
\begin{aligned}
\tilde{f}(\sigma(p)) & =\left[\int_{p_{0}}^{\sigma(p)} W\right]=\left[\int_{p_{0}}^{\sigma\left(p_{0}\right)} W+\int_{\sigma\left(p_{0}\right)}^{\sigma(p)} W\right]=v_{0}+\left[\int_{p_{0}}^{p} \sigma^{*} W\right] \\
& =v_{0}+\left[\int_{p_{0}}^{p} \bar{W}\right]=v_{0}+c \circ \tilde{f}(p) .
\end{aligned}
$$

Since $\sigma$ has order two, $\tilde{f}\left(p_{0}\right)=0=\tilde{f}\left(\sigma\left(\sigma\left(p_{0}\right)\right)\right)=v_{0}+c \circ \tilde{f}\left(\sigma\left(p_{0}\right)\right)$. Hence, $v_{0}=-c\left(v_{0}\right)$. This proves part (1) of the lemma and by a similar argument one can prove part (2).

Note that $\operatorname{Re}\left(v_{0}\right)$ may equal zero in which case it trivially has order-two in the group $\hat{A}(\tilde{M})$. Since $\tilde{\sigma}$ acts on $\hat{A}(M)$ by translation by the point $\operatorname{Re}\left(v_{0}\right)$ and $\sigma$ has order-two, it follows that everything in the kernel $K$ of $\bar{\pi}$ can be expressed as $k+r$, where $k \in T(\sqrt{-1})$, and $r$ is the image of a purely real 
vector in $\mathbf{C}^{k}$ and has order two in $A(\tilde{M})$. Since $r$ has order-two, $r=-r$ and so $c(k+4)=c(k)+c(r)=-k+r=-(k+r)$. This final equation completes the proof of the lemma.

We now describe a conformal property of certain special Riemannian nonorientable surfaces.

Definition 1.3. Consider $S^{2}$ to be $\mathrm{C} \cup\{\infty\}$ under stereographic projection and let $\delta: S^{2} \rightarrow S^{2}$ be the map induced by the complex conjugation. Let $\Gamma=\left\{p_{1}, \delta\left(p_{1}\right), \cdots, p_{k}, \delta\left(p_{k}\right)\right\}$ be $2 k$ distinct points in $\mathbf{C}$, let $P: \tilde{M}_{\Gamma} \rightarrow S^{2}$ be the 2-sheeted branched cover of $S^{2}$ branched over $\Gamma$, and let $\sigma: \tilde{M}_{\Gamma} \rightarrow \tilde{M}_{\Gamma}$ be the lift of $\delta: S^{2} \rightarrow S^{2}$ to $\tilde{M}_{\Gamma}$ which acts freely on $\tilde{M}_{\Gamma}$. A nonorientable Riemannian surface $M$ is called special if $M$ is conformally diffeomorphic to $\tilde{M}_{\Gamma} / \sigma$ for some choice of $\Gamma$. In particular, if $M$ is special, then the oriented 2 -sheeted cover of $M$ is hyperelliptic.

Theorem 1.4. Let $M$ be a closed Riemannian surface of negative Euler characteristic and let $f: M \rightarrow A(M)$ be the Albanese map. Then the following are equivalent:

(1) $f$ is not injective.

(2) There is a simple closed curve $\alpha$ on $M$ such that $f(\alpha)$ is a single point.

(3) $f$ is not an immersion.

(4) $M$ is a special surface.

Proof. Suppose that $M$ is special and that the oriented 2-sheeted cover of $M$ is $\tilde{M}_{\Gamma}$ for some choice of points $\Gamma$ in C. Let $h: \tilde{M}_{\Gamma} \rightarrow \tilde{M}_{\Gamma}$ be the hyperelliptic automorphism of $\tilde{M}_{\Gamma}$ which is the order-two covering transformation for the branched cover $P: \tilde{M}_{\Gamma} \rightarrow S^{2}$. Since the pull-back by $h$ of a harmonic 1-form on $\tilde{M}_{\Gamma}$ is the negative of that harmonic 1-form, the induced isometry $\bar{h}: A\left(\tilde{M}_{\Gamma}\right) \rightarrow A\left(\tilde{M}_{\Gamma}\right)=\mathbf{C}^{k} / \Lambda$ has linear part which is $-I$ where $I$ is the identity matrix. Directly from the definition of a special surface we conclude that

$$
\gamma=\operatorname{Fix}(h \circ \sigma)=P^{-1}(\mathbf{R} \cup\{\infty\}) \subset \operatorname{Fix}(\bar{h} \circ \bar{\sigma}),
$$

where $\operatorname{Fix}(h \circ \sigma)$ and $\operatorname{Fix}(\bar{h} \circ \bar{\sigma})$ are the fixed point sets of the respective automorphisms.

The above general discussion holds for any choice of the base point of $M_{\Gamma}$. Now choose $\tilde{p}_{0} \in \gamma$ and base point $p_{0}=\pi\left(\tilde{p}_{0}\right)$ for $M$. Let $\tilde{f}: \tilde{M}_{\Gamma} \rightarrow A\left(\tilde{M}_{\Gamma}\right)=$ $\mathbf{C}^{k} / \Lambda$ be the associated Albanese map and let $\hat{\gamma}=\tilde{f}(\gamma)$. Since $\tilde{p}_{0}$ is a fixed point of $\bar{h} \circ \bar{\sigma}$ and the linear part of $\bar{\sigma}$ is induced by complex conjugation (Lemma 1.2), $\bar{h} \circ \bar{\sigma}$ acts on $A\left(\tilde{M}_{\Gamma}\right)$ by $-c$ and hence $\bar{h} \circ \bar{\sigma}$ fixes $T(\sqrt{-1})$. Since $\hat{\gamma}$ must be fixed by $-c, T(\sqrt{-1})$ is a connected component of $\operatorname{Fix}(\bar{h} \circ \bar{\sigma})$ and $\hat{\gamma} \cap T(\sqrt{-1}) \neq \varnothing$, we conclude that $\hat{\gamma} \subset T(\sqrt{-1})$. In particular $\hat{\gamma}$ is in the kernel of $\bar{\pi}: A\left(\tilde{M}_{\Gamma}\right) \rightarrow A(M)$ and therefore $\bar{\pi}(\hat{\gamma})=f\left(p_{0}\right)=0$ is a single 
point. Hence part (4) of the theorem implies part (2). Clearly part (2) of the theorem implies parts (1) and (3). We shall complete the proof of the theorem by showing that part (1) implies part (3) and part (3) implies part (4). Let $f: M \rightarrow A(M)$ be the Albanese map for $M$.

Suppose that $f^{-1}(f(p))$ contains some point $q$ different from $p$, and choose the base point $p_{0}$ to be the point $p$. Let $\tilde{p}_{0}$ be one of the points in $\pi^{-1}\left(p_{0}\right)$ and let $\tilde{q}$ be one of the points in $\pi^{-1}(q)$. Let $\tilde{p}_{0}$ be the base point for $\tilde{M}$ and note that $\tilde{f}\left(\tilde{p}_{0}\right)$ and $\tilde{f}(\tilde{q})$ are both in the kernel of $\bar{\pi}$. Parts (1) and (3) of Lemma 1.2 immediately give

$$
\begin{gathered}
\tilde{f}(\sigma(\tilde{q}))=-\tilde{f}(\tilde{q})+v_{0}, \\
\tilde{f}\left(\tilde{p}_{0}\right)+\tilde{f}\left(\sigma\left(\tilde{p}_{0}\right)\right)=v_{0}, \\
\tilde{f}(\tilde{q})+f(\sigma(\tilde{q}))=v_{0} .
\end{gathered}
$$

(1.4) and (1.5) together with Abel's theorem show that $\tilde{p}_{0}+\sigma\left(\tilde{p}_{0}\right)-\tilde{q}-\sigma(\tilde{q})$ is the divisor of a meromorphic function $P: \tilde{M} \rightarrow S^{2}$ of degree-two. In particular if $h: \tilde{M} \rightarrow \tilde{M}$ is the order-two covering transformation for the branched cover $P$, then $h$ is the hyperelliptic automorphism on $\tilde{M}$ and $h$ extends to an isometry $\bar{h}$ on $A(\tilde{M})$ whose linear part is the negative of the identity matrix. Since $\bar{h} \circ \bar{\sigma}$ fixes the point $\tilde{p}_{0}, \bar{h} \circ \bar{\sigma}=-c$. Hence, the tangent space $T_{\tilde{p}_{0}} \tilde{M}$ is invariant under complex conjugation. Since $T_{\tilde{p}_{0}} \tilde{M}$ is invariant under complex conjugation, it must contain a purely imaginary vector. Thus the differential of $\bar{\pi} \mid \tilde{M}$ at $\tilde{p}_{0}$ has rank less than one, and part (1) implies part (3) of the theorem.

Suppose that $f$ is not an immersion at $p_{0}$ so that $T_{\tilde{p}_{0}} \tilde{M}$ contains a purely imaginary vector. Choose $p_{0}$ to be the base point of $M$ and choose a base point $\tilde{p}_{0}$ on $\tilde{M}$ that covers $p_{0}$. Since $T_{\tilde{p}_{0}} \tilde{M}$ is invariant under complex conjugation, part (1) of Lemma 1.2 implies that the Gauss or canonical map $G: \tilde{M} \rightarrow \mathbf{C} P^{k-1}$ has the same values at the points $\tilde{p}_{0}$ and $\sigma\left(\tilde{p}_{0}\right)$. Hence the canonical map of $\tilde{M}$ is not one-to-one. A classical theorem on Riemann surfaces [2] implies that $G(\tilde{M})$ is an embedded sphere $S^{2}$ contained in $\mathbf{C} P^{k-1}$ and that the induced map $\hat{G}: \tilde{M} \rightarrow S^{2}$ is a 2-sheeted branched cover. Part (1) of Lemma 1.2 also shows that $S^{2}$ is invariant under the automorphism $d: \mathbf{C} P^{k-1} \rightarrow \mathbf{C} P^{k-1}$ induced by complex conjugation. Furthermore $d \mid S^{2}$ has a fixed point at $G\left(\tilde{p}_{0}\right)$. Since the induced anticonformal map $d \mid S^{2}: S^{2} \rightarrow S^{2}$ has order-two and $d \mid S^{2}$ has a fixed point, $d \mid S^{2}$ is conjugate, under a conformal diffeomorphism of $S^{2}$ with $\mathrm{C} \cup\{\infty\}$, to the map induced by complex conjugation on C. Since $\sigma$ is a lift of $d \mid S^{2}$ to $\tilde{M}$ and acts freely on $\tilde{M}$, the definition of a special nonorientable surface shows that $M$ is special. This completes the proof of the theorem. 
Corollary 1. If the Albanese map of a closed nonorientable surface is a branched minimal immersion, then the map is a one-to-one immersion.

Proof. By Theorem 1.4 the corollary can only fail when the image of some curve on the surface composed with the Albanese map is a single point. Since this never occurs for a branched immersion, the corollary is proved.

Corollary 2. Suppose $M$ is a closed nonorientable Riemannian surface with $k>0$ independent harmonic 1-forms. Let $\tilde{\sigma}: \hat{A}(\tilde{M}) \rightarrow \hat{A}(\tilde{M})$ be the map defined in Lemma 1.2. Then $\tilde{\sigma}$ acts trivially if and only if $k$ is even. If $\Delta: \mathbf{R}^{k} \rightarrow A(M)$ denotes the universal cover of $A(M)$ where $M$ is not special, then $\Delta^{-1}(M)$ is a periodic embedded harmonic surface that is orientable when $k$ is odd and nonorientable when $k$ is even.

Proof. We first prove the corollary in the case where $M$ is not special. By Theorem 1.4, $M$ is a smooth embedded surface in $A(M)$. By Lemma $1.2, \tilde{\sigma}$ acts on $\hat{A}(\tilde{M})$ by translation by $\omega_{0}=\operatorname{Re}\left(v_{0}\right)$. If $\omega_{0}=0$, then the map $H: \hat{A}(\tilde{M}) \rightarrow A(M)$ is the identity map. When $\omega_{0} \neq 0$, then $\omega_{0}$ has order-two. If $\omega_{0} \neq 0$, then $\tilde{\sigma}$ acts on the embedded orientable surface $\operatorname{Re}(\tilde{f}(\tilde{M}))$ in $\hat{A}(\tilde{M})$ as an orientation reversing map. It remains to prove that $\omega_{0} \neq 0$ precisely when $k$ is odd. When $k$ is even, the classification of closed surfaces implies there is a Jordan curve $\gamma$ on $M$ whose regular neighborhood $N(\gamma)$ is of Möbius strip, $M-\gamma$ is an orientable surface and $\gamma$ represents the order-two element of the first homology group of $M$. Since the fundamental group of a torus is free abelian, then $f(\gamma)$ is homotopically trivial and hence the neighborhood $N(\gamma)$ lifts to $\Delta^{-1}(M)$. Since $\Delta^{-1}(M)$ contains a Möbius strip, it is nonorientable and so $\omega_{0}$ is zero.

When $k$ is odd it is easy to check that the image of $H_{1}(\tilde{M})$ in $H_{1}(M) /$ Torsion is a subgroup of index-two. Since $f_{*}: H_{1}(M) /$ Torsion $\rightarrow$ $H_{1}(A(M))$ is an isomorphism, there exists a 2-sheeted cover $\widetilde{A(M)}$ of $A(M)$ corresponding to the subgroup $f_{*}\left(\pi_{*}\left(H_{1}(\tilde{M})\right)\right) \subset H_{1}(A(M))$ for which $\pi$ 。 $\tilde{f}: M \rightarrow A(M)$ lifts but for which $f$ does not lift. It follows that $\omega_{0}$ is nonzero, $\widetilde{A(M)}=\hat{A}(\tilde{M})$ and $\Delta^{-1}(M)$ is orientable. This proves the corollary under the assumption that $M$ is not special.

If $M$ is special, then the last argument that $\tilde{\sigma}$ acts nontrivially on $\hat{A}(\tilde{M})$ works when $k$ is odd. If $k$ is even, then a similar analysis using the fact that the image of $H_{1}(\tilde{M})$ in $H_{1}(M) /$ Torsion is the entire group proves that $\bar{\sigma}$ acts trivially on $\hat{A}(\tilde{M})$. This completes the proof of the corollary.

Remark. Although it is not stated in Corollary 1 , it is easy to check that $T(\sqrt{-1})=\operatorname{Ker}(\bar{\pi})$ if and only if $k$ is even. When $k$ is odd, $T(\sqrt{-1})$ has index-2 in $\operatorname{Ker}(\bar{\pi})$. 


\section{References}

[1] P. Griffiths \& J. Harris, Principles of algebraic geometry, Wiley, New York, 1978.

[2] R. C. Gunning, Lectures on Riemann surfaces, Math. Notes, Vol. 6, Princeton University Press, Princeton, NJ, 1966.

[3] _ Lectures on Riemann surfaces: Jacobi varieties, Math. Notes, Vol. 12, Princeton University Press, Princeton, NJ, 1972.

[4] W. H. Meeks, III, The geometry and conformal structure of triply periodic minimal surfaces in $\mathbf{R}^{3}$, Doctoral Thesis, University of California at Berkeley, 1975.

[5] _ The conformal structure and geometry of triply periodic minimal surfaces in $\mathbf{R}^{3}$, Bull. Amer. Math. Soc. 83 (1977), 134-136.

[6] - The existence and geometry of triply periodic minimal surfaces, in preparation.

[7] _ 